\title{
ALGEBRAIC PROPERTIES OF THE SHIFT MAPPING
}

\author{
PATRICK DEHORNOY
}

(Communicated by Andreas Blass)

\begin{abstract}
We investigate the algebraic structure generated by the "shift" mapping $n \rightarrow n+1$ on $\mathbf{N}$ under composition and another operation defined below. This allows to prove results about certain free autodistributive structures.
\end{abstract}

1. A $J$-algebra will be the algebraic structure made by a set endowed with two binary operations $(x, y) \rightarrow x y$ and $(x, y) \rightarrow^{x} y$ satisfying the following identities - where ${ }^{x} y z$ stands for $\left({ }^{x} y\right) z$ and ${ }^{x y} z$ for ${ }^{(x y)} z$-:

$$
\begin{gathered}
x(y z)=(x y) z \\
x y={ }^{x} y x \\
x(y z)={ }^{x} y^{x} z \\
x y={ }^{x}\left({ }^{y} z\right) .
\end{gathered}
$$

It happens that $J$-algebras are rather natural and common structures: a $J$ algebra is nothing but a semigroup $M$ satisfying the weak commutativity condition

$$
(\forall x, y)(\exists z)(x y=z x)
$$

and such that the " $z$ " above can be chosen "functorially", i.e. in such a way that the mapping $x \mapsto[y \mapsto z]$ is in $\operatorname{Hom}(M$, End $M)$. In particular any semigroup satisfying (1) with an unique $z$ for every $x, y$ is a $J$-algebra (when ${ }^{x} y$ is defined to be this " $z$ ") and therefore every group becomes a $J$-algebra when endowed with the conjugacy operation ${ }^{x} y:=x y x^{-1}$.

Some properties of the $J$-algebras are quoted in [1] (where the $J$-algebras with an unit are called semi-Abelian monoids). In fact the interest for the $J$ algebras comes from set theory where, under some (very strong) large cardinal hypotheses, particular $J$-algebras can be obtained using elementary embeddings. It happens that certain properties of these elementary embeddings (that appear as basic tools in recent developments of set theory) originate in their structure

Received by the editors June 2, 1988 and, in revised form, November 28, 1988.

1980 Mathematics Subject Classification (1985 Revision). Primary 17A30, 17A50; Secondary 03 E55.

This work was partially supported by a CNRS grant PRC mathématiques \& informatique. 
of $J$-algebra [2], and this structure appears to be very rich and mysterious (see [4]). It is a natural conjecture to claim that the $J$-algebras associated to the elementary embeddings are free $J$-algebras (in the usual sense of algebra) and therefore a lot of questions arise about the free $J$-algebras and, specially, the free monogenic one. However it is easy to describe the free $J$-algebras as quotients of free magmas (i.e. sets of terms) under convenient equivalences [1], [3] and this gives a way for proving that certain identities do hold in these structures. But it is difficult to prove that an identity does not hold, i.e. to prove that particular terms are not equivalent.

The usual way for proving that two terms $t_{1}, t_{2}$ are not equivalent is to find a particular $J$-algebra $\mathfrak{F}$ and a projection $\pi$ onto $\mathfrak{F}$ such that $\pi\left(t_{1}\right)$ and $\pi\left(t_{2}\right)$ are not equal. So in order to study the case of the free monogenic $J$ algebra, we have to exhibit interesting monogenic $J$-algebras. In fact, we are interested in terms involving the second operation $(x, y) \rightarrow{ }^{x} y$, and therefore the $J$-algebras quoted above, in particular those associated to groups, cannot be helpful as the corresponding operation is trivial: every monogenic semigroup is Abelian, and so ${ }^{x} y$ is $y$ for all $x, y$. So we need monogenic $J$-algebras with nontrivial operation ${ }^{x} y$. Of course, the $J$-algebra constructed in set theory is a natural candidate, but the problem is that we wanted to study the free $J$-algebras first because the set theoretic one is difficult to handle with, and moreover the very existence of this $J$-algebra is hypothetic. Some possibility is to exhibit finite projections of the set theoretic $J$-algebra: because they are finite, their properties do not depend on any set theoretic hypothesis. This approach is possible (see the proof of Lemma 5), but up to now only three $J$-algebras have been described in this way such that the corresponding substructures generated by the operation ${ }^{x} y$ alone have 2,4 and 8 elements. This does not solve our questions, and we have to find another way for constructing monogenic $J$-algebras: the aim of this note is to provide such a construction.

2. In order to describe new $J$-algebras, we use families of mappings of one set into itself. If $f$ is a mapping of $X$ into itself, we write $\operatorname{Im} f$ for the image of $f, \operatorname{coIm} f$ for $X \backslash \operatorname{Im} f$ and Fix $f$ for $\{x \in X ; f(x)=x\}$.

Definition. For every set $X$, let $\mathfrak{F}_{x}$ be the set of all one-one mappings of $X$ into itself; we endow $\mathfrak{F}_{x}$ with the composition operation:

$$
f g(x):=f(g(x))
$$

and with the following operation:

$$
{ }^{f} g(x):= \begin{cases}f g f^{-1}(x) & \text { if } x \text { is in } \operatorname{Im} f \\ x & \text { if } x \text { is in } \operatorname{colm} f .\end{cases}
$$

Proposition. $\mathfrak{F}_{x}$ is a $J$-algebra.

Proof. Elementary; for instance, let us verify that ${ }^{f g} h={ }^{f}\left({ }^{g} h\right)$ holds; let $x$ be any member of the set $X$. 
Case 1. $x$ is in $\operatorname{coIm} f$. Then $x$ is in $\operatorname{coIm}(f g)$, so we get:

$$
{ }^{f g} h(x)=x={ }^{f}\left({ }^{g} h\right)(x) \text {. }
$$

Case 2. $x$ is in $f[\operatorname{coIm} g]$. Then $x$ is in $\operatorname{coIm}(f g)$, and $f^{-1}(x)$ is in coIm $g$, so we get:

$$
{ }^{f g} h(x)=x \text { and }{ }^{f}\left({ }^{g} h\right)(x)=f^{g} h f^{-1}(x)=f f^{-1}(x)=x .
$$

Case 3. $x$ is in $\operatorname{Im}(f g)$. Then $f^{-1}(x)$ is in $\operatorname{Im} g$, so we get:

$$
{ }^{f g} h(x)=f g h g^{-1} f^{-1}(x) \text { and }{ }^{f}\left({ }^{g} h\right)(x)=f^{g} h f^{-1}(x)=f g h g^{-1} f^{-1}(x) .
$$

3. Of course, if $X$ is a finite set, then the $J$-algebra $\mathfrak{F}_{x}$ is nothing but the symmetric group $\mathfrak{S}_{X}$ endowed with the conjugacy operation. The smallest interesting case is therefore the case of $\mathfrak{F}_{\mathbf{N}}$. In the sequel, the shift operation on $\mathbf{N}$,

$$
n \mapsto n+1
$$

will be denoted by $s$, and we let $\mathfrak{D}$ be the sub- $J$-algebra of $\mathfrak{F}_{\mathbf{N}}$ generated by $s$. So $\mathfrak{D}$ is a monogenic $J$-algebra, and it is easily seen that it is nontrivial with respect to ${ }^{x} y$ (see 4 below). Our first observation will be that $\mathfrak{D}$ is essentially the only monogenic sub- $J$-algebra of any $\mathfrak{F}_{x}$. If $G$ is a group, we shall denote by $\mathfrak{c}(G)$ the $J$-algebra obtained by adding the conjugacy operation to $G$.

Proposition. Let $X$ be any set; the monogenic sub-J-algebras of $\mathfrak{F}_{x}$ can only be of the following types: $\mathfrak{D}, \mathfrak{D} \times \mathfrak{c}(\mathbf{Z} / n \mathbf{Z}), \mathfrak{D} \times \mathfrak{c}(\mathbf{Z}),\{1\}, \mathfrak{c}(\mathbf{Z} / n \mathbf{Z}), \mathfrak{c}(\mathbf{Z})$.

Proof. First we notice that, if $\left\{X_{1}, X_{2}\right\}$ is a partition of $X$, and $f, g$ are in $\mathfrak{F}_{X}$ such that $X_{1}, X_{2}$ are closed under $f$ and $g$, then $X_{1}, X_{2}$ are closed under $f g$ and ${ }^{f} g$ as well and, for $i=1,2, f g \mid X_{i}$ (resp. $\left.{ }^{f} g \mid X_{i}\right)$ is $\left(f \mid X_{i}\right)\left(g \mid X_{i}\right)$ (resp. ${ }^{f \mid X_{i}} i g \mid X_{i}$ ). So, if $f$ is as above, the sub- $J$-algebra of $\mathfrak{F}_{X}$ generated by $f$ is the product of the sub- $J$-algebras of $\mathfrak{F}_{X_{1}}, \mathfrak{F}_{X_{2}}$ generated by $f\left|X_{1}, f\right| X_{2}$, respectively. Now for $f$ in $\mathfrak{F}_{X}$, define a sequence $\left\langle\operatorname{Im}_{p}(f) ; p \in \mathbf{N} \cup\{\infty\}\right\rangle$ as follows:

$$
\begin{aligned}
\operatorname{Im}_{O}(f) & :=\operatorname{coIm} f \\
\operatorname{Im}_{p+1}(f) & :=f\left[\operatorname{Im}_{p}(f)\right] \text { for } p \text { in } \mathbf{N} \\
\operatorname{Im}_{\infty}(f): & =X \backslash \cup \operatorname{Im}_{p}(f) .
\end{aligned}
$$

For $p>0$, the set $\operatorname{Im}_{p}(f)$ is included in $\operatorname{Im} f$, so $\operatorname{Im}_{O}(f)$ and $\operatorname{Im}_{p}(f)$ are disjoint. As $f$ is one-one, it follows that the sets $\operatorname{Im}_{p}(f)$ and $\operatorname{Im}_{q}(f)$ are disjoint for any $p, q$ in $\mathbf{N}$, and $\left\langle\operatorname{Im}_{p}(f) ; p \in \mathbf{N} \cup\{\infty\}\right\rangle$ is a partition of $X$. Clearly the sets $\operatorname{Im}_{\infty}(f)$ and $X \backslash \operatorname{Im}_{\infty}(f)$ are closed under $f$, and therefore the subalgebra generated by $f$ is the product of the subalgebras generated by $f \mid \operatorname{Im}_{\infty}(f)$ and $f \mid X \backslash \operatorname{Im}_{\infty}(f)$. As $\operatorname{Im}_{O}\left(f \mid \operatorname{Im}_{\infty}(f)\right)$ and $\operatorname{Im}_{\infty}\left(f \mid X \backslash \operatorname{Im}_{\infty} f\right)$ are empty, we are reduced to study separately two cases. We write $\langle f\rangle$ for the sub- $J$-algebra generated by $f$. 
Case 1. $\operatorname{Im}_{o}(f)$ is empty, i.e. $f$ is onto. An immediate induction shows that any member of $\langle f\rangle$ is onto, so $\langle f\rangle$ is $\mathfrak{c}(G)$ where $G$ is the subgroup of $\mathfrak{S}_{X}$ generated by $f$. This subgroup (if not trivial) is isomorphic to $\mathbf{Z}$ or to some $\mathbf{Z} / n \mathbf{Z}$ (and as these groups are Abelian the corresponding conjugacy operation is the trivial one: ${ }^{x} y=y$ ).

Case 2. $\operatorname{Im}_{\infty}(f)$ is empty. We know that, for every $p$ in $\mathbf{N}, f \mid \operatorname{Im}_{p}(f)$ is a bijection of $\operatorname{Im}_{p}(f)$ onto $\operatorname{Im}_{p+1}(f)$. Let $g$ be any member of $\langle f\rangle$; we define, when possible, a member $\dot{g}$ of $\mathfrak{D}$ as follows:

$$
\dot{g}(p)=q \text { iff } g\left|\operatorname{Im}_{p}(f)=f^{q-p}\right| \operatorname{Im}_{p}(f)
$$

(where $f^{n}$ stands for $f \cdots f, n$ terms, if $n>0$, for id if $n$ is 0 , and for $f^{-1} \cdots f^{-1},|n|$ terms, if $\left.n<0\right)$. If $\dot{g}$ exists, it completely determines $g$ (as $\operatorname{Im}_{\infty}(f)$ is empty), so $g \rightarrow \dot{g}$ is one-one. Next $\dot{f}$ exists and is equal to $s$. In order to prove that $g \rightarrow \dot{g}$ is an isomorphism of $\langle f\rangle$ onto $\mathfrak{D}$, it suffices to show that, if $\dot{g}$ and $\dot{h}$ are defined, then $(g h)^{\circ}$ and $\left({ }^{g} h\right)^{\circ}$ are defined and equal, respectively, to $\dot{g} \dot{h}$ and ${ }^{\dot{g}} \dot{h}$. This is easy to verify by an explicit computation.

The proposition then follows by pasting the various possible cases.

Remark. It follows that every monogenic subalgebra of $\mathfrak{D}$ is isomorphic to $\mathfrak{D}$, and therefore any identity involving a single member say $f$ of $\mathfrak{D}$ is true if, and only if, the corresponding identity is true for $s$.

4. Various combinatorial properties of the members of $\mathfrak{D}$ can be established (using inductions on the two operations in $\mathfrak{D}$ ). For instance, there exist two mappings $\mu, \nu: \mathfrak{D} \rightarrow \mathbf{N}$ such that, for every $f$ in $\mathfrak{D}$, the following holds:

$$
(\forall n \geq \nu(f))(f(n)=n+\mu(f)),
$$

and the following equalities hold:

$$
\mu(f g)=\mu(f)+\mu(g), \quad \mu\left({ }^{f} g\right)=\mu(g) .
$$

However the property of being eventually equal to an iterated shift does not characterize the members of $\mathfrak{D}$ : indeed one can prove that, for $f$ in $\mathfrak{D}$, either 0 is $f(0)$ or 0 is in $\operatorname{coIm} f$, and therefore the mapping $f$ such that $f(0)$ is $1, f(1)$ is 0 and $f(n)$ is $n+1$ for $n \geq 2$ is not in $\mathfrak{D}$. We shall use $\mathfrak{D}$ to study the free $J$-algebras; consider the following natural questions (the conjectured answers are positive in the three cases):

Questions. Assume that $\mathfrak{F}$ is a free $J$-algebra and $x, y, z$ are in $\mathfrak{F}$ :

(i) is ${ }^{x} y$ always distinct of $x$ and of $y$ ?

(ii) does ${ }^{z} x={ }^{z} y$ imply $x=y$ ?

(iii) does ${ }^{x} z^{y} z={ }^{y} z^{x} z$ imply $x=y$ when $z$ is a generator of $\mathfrak{F}$ ?

We get the following partial answers:

Proposition. Assume that $\mathfrak{F}$ is a free $J$-algebra; for $x, y, z$ in $\mathfrak{F}$, we have:

(i) $x \neq^{x} y$ and $y \neq^{x} y$; 
(ii) ${ }^{z} x={ }^{z} y$ implies $\pi(x)=\pi(y)$ for every homomorphism $\pi$ of $\mathfrak{F}$ to $\mathfrak{D}$;

(iii) ${ }^{x} z^{y} z={ }^{y} z^{x} z$ implies $\pi(x)=\pi(y)$ for every homomorphism $\pi$ of $\mathfrak{F}$ to $\mathfrak{D}$ such that $\pi(z)$ is $s$ (if any).

The proof is immediate from the following:

Lemma. For every $f, g, h$ in $\mathfrak{D}$, we have:

(i) $f \neq{ }^{f} g$ and $g \neq{ }^{f} g$;

(ii) ${ }^{h} f={ }^{h} g \Rightarrow f=g$;

(iii) ${ }^{f} g={ }^{g}{ }^{f} s \Rightarrow f=g$.

Proof. (i) No member in $\mathfrak{D}$ can be onto as $\operatorname{coIm}(f g)$ includes $f[\operatorname{coIm} g]$; now if $n$ is in $\operatorname{coIm} f, n$ is in $\operatorname{Im}\left({ }^{f} g\right)$, so ${ }^{f} g$ cannot be $f$. On the other hand, $\operatorname{Fix}\left({ }^{f} g\right)$ is $f[$ Fix $g] \cup \operatorname{coIm} f$ so $\operatorname{Fix}\left({ }^{f} g\right)$ cannot be equal to $\operatorname{Fix}(g)$, since the first set has strictly more elements than the second one has and both are finite because of the existence of the function $\nu$, above (and the fact that the values of $\mu$ are $>0$ ). So ${ }^{f} g=g$ is impossible.

(ii) Easy: assume ${ }^{h} f={ }^{h} g$; then we get: ${ }^{h} f h={ }^{h} g h$, i.e. $h f=h g$, hence $f=g$, as $h$ is one-one.

(iii) Assume that ${ }^{f} s$ and ${ }^{g} s$ commute. First we show that $\operatorname{Im} f$ and $\operatorname{Im} g$ are equal. As $\operatorname{Fix}(s)$ is empty, $\operatorname{Fix}\left({ }^{f} s\right)$ is exactly $\operatorname{coIm} f$. Assume that $g(n)$ is in $\operatorname{coIm} f$, hence in $\operatorname{Fix}\left({ }^{f} s\right):{ }^{f} s^{g} s(g(n))$ is ${ }^{f} s(g(n+1))$, while ${ }^{g} s^{f} s(g(n))$ is ${ }^{g} s(g(n))$, i.e. $g(n+1)$. So $g(n+1)$ is in $\operatorname{Fix}\left({ }^{f} s\right)$ as well, and we can iterate, concluding that $\operatorname{Fix}\left({ }^{f} s\right)$ is infinite, a contradiction. So $\operatorname{Im} g$ is included in $\operatorname{Im} f$, and, symmetrically, $\operatorname{Im} f$ is included in $\operatorname{Im} g$. Now let $h$ be $g^{-1} f$ : $h$ is a bijection of $\mathbf{N}$. Let $n$ be any integer: an easy computation shows that ${ }^{f} s^{g} s(f(n))$ and ${ }^{g} s^{f} s(f(n))$ are, respectively, $f s h^{-1} s h(n)$ and $g s h s(n)$, so the hypothesis implies $s k=k s$ where $k$ is $h^{-1} s h$. As coIm $s$ has one member, $\operatorname{coIm} k$ has one member, and therefore the condition $s k=k s$ (i.e. $k(n+1)=k(n)+1$ for all $n)$ implies $k=s$. So we have $s h=h s$ and $\operatorname{coIm} h$ is empty: we conclude that $h$ is the identity, i.e. $f$ and $g$ are equal.

Remark. The results above are incomplete in (ii) and (iii) as we said that the conjectured conclusion is $x=y$ rather than $\pi(x)=\pi(y)$ (under the hypothesis that $z$ is a generator of $\mathfrak{F}$ in (iii)). This of course could be equivalent (in the monogenic case) if $\mathfrak{D}$ were free, but this is not true, as quoted below. Nevertheless a complete answer in $\mathfrak{F}$ can be obtained by a direct verification for particular occurrences of these questions. For instance assume that $a$ is one of the generators of $\mathfrak{F}$ : there exists an homomorphism $\pi$ of $\mathfrak{F}$ onto $\mathfrak{D}$ such that $\pi(a)$ is $s$. Now, if $p, q$ are distinct integers $\pi\left(a^{p}\right)$ and $\pi\left(a^{q}\right)$ are certainly different as $s^{p}$ and $s^{q}$ are, so we can conclude that ${ }^{a^{p}} a$ and ${ }^{a^{q}} a$ do not commute in $\mathfrak{F}$.

5. Lemma. $\mathfrak{D}$ is not a free $J$-algebra. 
Proof. Write (in any $J$-algebra) $x^{\prime}$ for ${ }^{x} x$ and $x^{\prime \prime}$ for $\left(x^{\prime}\right)^{\prime}$. It is easy to verify that the following equality holds in $\mathfrak{D}$ :

$$
s^{\prime \prime} s^{\prime}={ }^{s^{\prime}} s s^{\prime} .
$$

We claim that this equality is false in the free $J$-algebra generated by $s$. In order to prove that, it suffices to exhibit a $J$-algebra $\mathfrak{F}$ and an element $x$ of $\mathfrak{F}$ such that:

$$
x^{\prime \prime} x^{\prime} \neq{ }^{x^{\prime} x} x^{\prime} .
$$

(if the equality were true in the free $J$-algebra it would be true in any $J$-algebra). But consider on the set $\{0,1,2,3\}$ the binary operation $(x, y) \mapsto{ }^{x} y$ whose

\begin{tabular}{|c|c|}
\hline & 0123 \\
\hline 0 & 2233 \\
\hline 1 & 3333 \\
\hline 2 & 1133 \\
\hline 3 & $\begin{array}{llll}0 & 1 & 23\end{array}$ \\
\hline
\end{tabular}
table is as follows:

This table has been constructed as a projection of the (hypothetic) $J$-algebra associated to an elementary embedding. It is easy to verify that the identity

$$
{ }^{x}\left({ }^{y} z\right)={ }^{x_{y}}\left({ }^{x} z\right)
$$

holds in this structure. Now it is shown in [1] that every such structure can be extended to a $J$-algebra. Let $\mathfrak{F}$ be the $J$-algebra associated in this way. In $\mathfrak{F}$ we verify:

$$
0^{\prime \prime} 0^{\prime}={ }^{3} 2=2 ; \quad{ }^{0^{\prime}} 0^{\prime}={ }^{1} 2=3 .
$$

This is enough to conclude.

It is an open question to describe all relations that are true in $\mathfrak{D}$ but not in the free monogenic $J$-algebra, i.e. to describe completely the canonical projection of the free monogenic $J$-algebra onto $\mathfrak{D}$.

6. We conclude this note by showing how certain equations in the free $J$-algebra can be solved using $\mathfrak{D}$. Assume that $\mathfrak{F}$ is the free $J$-algebra generated by $a$. We consider equations of the following type

$$
x^{(p)}=a^{(q)},
$$

where $p, q$ are fixed integers and $x^{(p)}$ is defined by: $x^{(0)}:=x, x^{(p+1)}=$ $x^{(p)^{\prime}}$. These equations are natural and interesting because every element of $\mathfrak{F}$ is solution of one (in fact infinitely many) such equation: this property is true in every $J$-algebra, namely if $b$ is in the subalgebra generated by $a$, there 
exist $p, q$ such that $b^{(p)}$ is equal to $a^{(q)}$. Consider for instance the following equation in $\mathfrak{F}$ :

$$
x^{\prime}=a^{\prime \prime}
$$

Assume that $x$ is a solution, and let $f$ be $\pi(x)$ where $\pi$ is the projection of $\mathfrak{F}$ onto $\mathfrak{D}$ such that $\pi(a)$ is $s$. We get:

$$
f^{\prime}=s^{\prime \prime} \text {. }
$$

As coIm $g^{\prime}$ is always equipotent to coIm $g$, certainly coIm $f$ must have exactly one element, say $q$. Now $f^{\prime}(q)$ is $q$, so $q$ must be in $\operatorname{Fix}\left(s^{\prime \prime}\right)$, that is $\{0,1\}$. For $n \geq 2, f(n)$ is therefore equal to $f^{\prime}(n)$, hence to $s^{\prime \prime}(n)$, i.e. to $n+1$. So $f$ is either $s^{\prime}$ or ${ }^{\prime} s$. We are reduced to solve:

$$
\pi(x)=s^{\prime}
$$

and

$$
\pi(x)={ }^{s^{\prime}} s .
$$

The general solution of such equations is unknown, but in the actual case it is easy to solve, for $\operatorname{Fix}\left(s^{\prime}\right)$ and $\operatorname{Fix}\left({ }^{s^{\prime}} s\right)$ have only one element, and this implies that $x$ must be ${ }^{y} a$ for some $y$ such that $\pi(y)$ is, respectively, $s$ or $s^{\prime}$. Finally this proves that $y$ is $a$ or $a^{\prime}$, and we conclude that $x=a^{\prime}$ and $x={ }^{a^{\prime}} a$ are exactly the solutions of the equation $x^{\prime}=a^{\prime \prime}$ in $\mathfrak{F}$. Giving the full details entails a more precise study of the free $J$-algebra, which appears to be a rich subject but is outside the scope of this note (see [3]).

\section{REFERENCES}

1. Patrick Dehornoy, Infinite products in monoids, Semigroup Forum 34 (1986), 21-68.

2. 257-287.

3. __ Free distributive groupoids, J. Pure Appl. Algebra (to appear).

4. Randall Dougherty, A note on critical points of elementary embeddings, Notes.

5. Jozef Dudek, Some remarks on distributive groupoids, Czechoslovak Math. J. 31, 106 (1981), 451-456.

6. David Joyce, A classifying invariant of the knot, the knot quandle, J. Pure Appl. Algebra 23 (1982), 37-66.

7. _ـ Simple quandles, J. Algebra 79 (1982), 307-318.

8. Tomas Kepka, Notes on left-distributive groupoids, Acta Univ. Carolin.-Math. Phys. 22 (1981), 23-37.

9. Nabuo Nobusawa, A remark on conjugacy classes in simple groups, Osaka J. Math. 18 (1981), 749-754.

10. R. S. Pierce, Symmetric groupoids, Osaka J. Math. 15 (1978), 51-76 \& 16 (1979), 317-348.

11. Sherman Stein, Left distributive quasigroups, Proc. Amer. Math. Soc. 10 (1959), 577-578.

Defartement de Mathématiques, Université de Caen, 14032, Caen, France 